\title{
Complexity theory in management of heritage architecture preservation
}

\author{
A. A. Berezin ${ }^{1} \&$ V. V. Gridin ${ }^{2}$ \\ ${ }^{I}$ Department of Engineering Physics, McMaster University, Canada \\ ${ }^{2}$ Department of Chemistry, Technion-Israel Institute of Technology, \\ Israel
}

\begin{abstract}
The preservation of heritage architecture (PHA) is a complex socio-economic enterprise that transpires space, time and cultural settings. Thus, management and decision making in PHA proceeds within a variety of political and economic pressures from different groups with their own vested interests. Yet another aspect is brought by deleterious physical and environmental factors. The present paper discusses PHA in its practical management within the Theory of Complex Systems and some ideas of modern physics.

Keywords: heritage architecture, management, complexity theory, decision making, virtual reality, fuzzy logic, quantum logic, symmetry breaking.
\end{abstract}

\section{Introduction}

Preservation of heritage architecture (PHA) is one of several major activities in maintaining cultural and intellectual legacy for the posterity. As such, it is one of the central moral duties of any genuine civilization. It involves the whole gamut of various aspects, a mere listing of which can take several pages. Preservation of all kinds of literature (fictional narratives, scientific, philosophical, religious and mythological text and artefacts, etc), numerous forms of visual arts (paintings, graphics, woodcuts, sculptures, etc), photographs and cinematography, applied arts and musical performances, technological artefacts (now collected in numerous science museums) and, finally, heritage architecture (HA) - this is just a short list (by far incomplete) of items falling under the definition of heritage. 
Regretfully, the overall record of how civilizations manage and preserve their heritage is far from excellent. Accidental and intentional destruction of many valuable items in the above collection is a common happening. Such events as, e.g., burning of great libraries of antiquity, desecration and destruction of temples and iconic art, demolition of older buildings having architectural value these are all well known examples of human negligence, indifference and at times - hostility - to their heritage. Preservational efforts - while often also significant and praiseworthy - are often questioned, objected and challenged by various power-holders and vested interests.

This is of special concern for highly unusual "off-line" architectural constructs designed by such original visionaries as, e.g. Gaudi (Spain), Le Corbusier (France), or Oscar Niemeyer (Brazil) and scores of less famous. Likewise, some segments of society may consider this-or-that object of HA objectionable for a variety of reasons and even lobby for its destruction. The said interaction of numerous (and often conflicting) factors makes the analysis of practices of PHA a proper case for some methods of physical sciences, in particular the Theory of Complex Systems (TCS).

Common to various versions of TCS in physical, engineering, bioevolutionary and managerial areas is a trend to reduce multi-parametric problem to a model with only a few parameters. When such simplification succeeds, it provides handy tools for decision-making by some reductionistic techniques (e.g., fuzzy logic, neural networks, etc). Since almost all executive decisions are reducible to chains of YES/NO choices, such a strategy calls for a visionary modelling to find an optimal solution. Due to the fact that PHA is a societal issue, its strategy and managerial decisions are subjected to a gamut of political, economical and pragmatic pressures and often highly affected by the dominant Zeitgeist of the day.

Literature references in this article are minimal because the massive use of the Internet search engines by key words makes extensive formal bibliographies largely obsolete. This phenomenon quickly becomes virtually universal in the majority of research areas. In almost all cases "Googling" the key names and/or concepts instantaneously brings up a wealth of information. As such, this "paradigm shift" itself calls for its own analysis and philosophical reflection, e.g., blurring the concept of individual authorship and some dilution of the ideas of legal copyright which are recently become especially evident in connection with rampant downloads of music - a phenomenon which for all practical purposes remains virtually uncontrollable.

\section{General comments}

Present article discusses some conceptual aspects related to PHA activities such as priority setting and overall management of PHA. The authors, who are physicists, are looking at PHA issues from the vista of physical metaphors and analogies. Presently, methods and concepts of physical sciences are productively used in a variety of other areas of human activity [1]. Examples of the latter can be found is such diverse areas as economics and marketing, health sciences, 
ecology, demographics, management, decision making, and even in such areas as romantic relationships and sexology. Likewise, there are significant and growing literature on relationships between physics and visual arts [2] to which architecture is closely related.

\subsection{History and heritage}

As any other area of human interest, drive to preserve cultural heritage has mixed history and includes many aspects. It remains so for all aspects of legacy and heritage coming from previous generations - architectural, artistic (visual arts, sculptures), technical artefacts (e.g., older optical instruments or type-writers) which are now kept in museums, as well as literature, performance arts, etc.

\subsubsection{Ups and downs of preservation}

History not always played (or plays) well on a side of PHA. More often than not particular cultures where primarily interested in maintenance of artefacts produced by these same cultures. After the collapse of the Roman Empire there were no major or systematic efforts to preserve Roman or Greek architecture. During the medieval period things were largely left on their own to erode and disintegrate. The fact that certain samples or architectural marvels (some Roman viaducts, bridges, temples, etc) still reached our times was mostly due to their initial durability and quality of construction rather than any specific targeted efforts to preserve them for the posterity. And even those constructs of antiquity which have survived to our days have often reached us in pieces and fragments rather than in their original totality and integrity.

\subsubsection{Private and communal property}

In the majority of human civilizations there is a demarcation between private and communal property. Most of residential and business buildings - what is normally presently labelled under the term "real estate" - exist in a form of private property owned by either individuals or corporations. In this regard, there is considerable latitude in what can be understood as a "corporation" in this context; for example, cathedrals and temples normally owned by religious organizations or congregations. Their legal rights to the buildings (for examples, making changes to the building) may often be subjected to limitations in those cases when such buildings are designated as architectural heritage. Some other types of architectural constructs (e.g., bridges) may technically belong to municipalities or owned by states or governments. This issue, however, is prone to various grey areas and cases of mixed identity. A vivid example is an architectural marvel known as Brooklyn Bridge.

\subsection{3 "Sell the bridge" scams}

The broadly known expression "I have a bridge for sale" comes from the story of the Brooklyn Bridge in New York City. At its completion (1883) it was the longest suspension bridge in the world. From the time its construction has started and after it was opened, it was an object of a rivalry and dispute between boroughs of Manhattan and Brooklyn as to who, actually, owns the bridge. 
Uncertainly of the situation lead to several scams to "sell" the bridge to private hands. Presently Brooklyn Bridge is a public property and an item of the protected architectural heritage, having high historical and technological value, as well as impressive folkloristic and legendary significance. There are some similar stories with other publicly used facilities. They demonstrate that from the side of PHA interests, the issues of ownership can complicate the situation in terms of the decision making (e.g., how to repair or renovate), priority setting and designation of an item as a protected historical heritage.

\subsubsection{Individual versus collective decision making}

While some names of the ancient architects have reached our times, the scores of others did not make it to today. Some larger constructs as, for example, the Great Wall of China, may not have a single individual behind them. And yet from Giotto, Brunelleschi and Bernini to Gaudi, Le Corbusier and Niemeyer the majority of architectural creations are usually the result of the individual creativity. This somewhat changes nowadays when the Virtual Reality and computer packages become norm of the operation. Often in architectural firms many individuals contribute to various stages of the design of a particular project. As in sciences, where most of research papers are presently written in multiple co-authorships (the records go to up to 500 authors names on a single paper!), many architectural items are designed collectively.

Similar erosion of individuality comes with the modern trend of collective decision making. This is a contentious issue. On one hand, it looks almost as a trivial truth that "several heads in better than one". People, according to this thesis, can pool up their expertise and insights and arrive to better decisions. And often this is the case. But often the opposite is the case and the history is full of stories when the collective ("democratic") decisions led to spectacular blunders. In a historical hindsight it is worthy to notice that in the feudal system most architectural decisions were made by powerful individuals. This is why samples of magnificent architecture have reached us from Medici's Florence. It is doubtful that the modern corporate Board of Directors would approve construction of Florence Dome ("waste of money") or Giotto's Campanile (unless there will be commercial restaurant on top, like on Toronto's CN Tower).

\subsubsection{Culture of consumerism and critical decisions}

Regardless of possible best intentions, the thinking process of many people today is moulded in the environment of massive consumer culture, rampant (over)supply of goods and services [3], non-stop advertisement, glorification of shopping - and, at the same time, constant (and usually phony) teasers of "savings". As popular cliché goes, Americans care not so much what they spend, but how much they "save" [3]. Paranoia of (illusionary) "savings", which is implanted into the society by brainwashing omni-present multi-billion advertisement industry, contaminates minds of people from cradle to grave. Only relatively few individuals, capable of thinking outside the box, are capable to truly resist it. But in general, this whole realm of existence within the consumerism affects people's ability to produce optimal decisions, especially when the environment of collective (board) decision making dilutes the 
individual responsibility. This may be especially perilous for PHA decisions when it comes to highly original and unusual ("off-mainstream") architectural creations.

\subsubsection{Original and unusual architecture}

Many distinguished architects are mostly known for their high level of originality. Talking only about modern times, one can mention Antoni Gaudi (1852-1926), Frank Lloyd Wright (1867-1959), Le Corbusier (1887-1965), or Oscar Niemeyer (b. 1907). Like in surrealistic painting of Salvador Dali (who, by the way, also produced some architectural designs), these masters often use unusual combination of structural elements and decorative additions to make their architectural creations aesthetically appealing. The problem is that such aspects as aesthetic appeal do not, in general, have common acceptance among the public. Tastes and evaluation criteria of people are vastly different and what some see as exciting and uplifting, others may see as odd and irrelevant. Likewise, there are often significant disagreements as to whether this-or-that building or construction fits the surroundings in which it is located (e.g., cities or nature) and hence, the issues of their preservation and maintenance may become a crux of the conflict. This brings a yet another aspect to PHA decisions and subsequent activities.

\subsection{Physical analogies as thinking tools}

From the review of the effects of modern society on human psychology and the decision making process, we now turn to the discussion of usefulness of physical analogies and metaphors in the activities related to PHA and heritage preservation in general. Due to proven helpfulness of physical models in many over areas, as it was mentioned above, the authors of this paper propose a view that the incorporation of physical concepts into PHA strategies may drastically enhance the quality of PHA and leave the posterity with a richer and better preserved architectural heritage.

\subsubsection{Physical and every-day thinking}

These authors are far from claiming that physicists are somehow "above" the common society or that their brains are somehow "superior". Even greatest physicists of all times, such as Newton or Einstein, have demonstrated an utter humility as to their own ability to comprehend the world as their numerous quotations humbly attest. And yet, a mode of thinking of physicists and their years of training and subsequent active work in the field renders some specific trends which in other areas of human endeavour may be far less pronounced and even less so utilizable.

\subsubsection{Revolutions in physics and architecture}

Both physics and architectural styles experience cyclical "revolutions". There is, however, a radical difference between both. We all know about such "revolutions" in physics as Relativity Theory and Quantum Physics or, more recently, Theory of Chaos, or novel ideas on structure of space and time. Such 
ideas as superstrings or Quantum Potential (David Bohm) look none but revolutionary. However, according to Thomas Kuhn [4], revolutions in science go as "paradigm shifts". They do not normally deny or discard previous stages (or pervious theories), but incorporate them as partial cases or approximations. All theories remain at service. Classical Thermodynamics, which neglects atomic structure of matter and views heat (thermal energy) as a continuous substance, was not replaced by Statistical Physics which is based on the explicit picture of microscopic (atomic) states. Both these theories remain useful for their own purposes. There is no internal contradiction between them. Likewise, Classical Mechanics can be seen as a partial (asymptotic) case of Relativity Theory or Quantum Mechanics. All these theories are highly useful in their own realms.

Not so in architecture. Here the scale of values is much less fixed and changes with time. Some architectural styles may go out of fashion and become targets of public dislike. Some samples of these styles may be slotted for demolition, especially when they are located in "inconvenient" areas where the pressure of new (usually, commercial) development is high. The same is also the case for such places as cemeteries which often have good pieces of miniarchitecture like family crypts. To preserve them, especially in the environment of growing cities, may often turn out an insurmountable task. This additional challenge for PHA management which in this case, apart from the architecture in a proper meaning, also related to the preservation of folkloristic and community legacy, not to mention issues of ethical sensitivity.

\subsubsection{Physics as conservative science}

It may sound odd and even weird to state that physics is a conservative science. And yet, the first thing anyone faces at first exposure to physics is the encounter with the conservation laws. Energy and Momentum conservation is the cornerstone of Newtonian physics. Einstein's law of the mass-energy equivalence (famous $\mathrm{E}=\mathrm{mc}^{2}$ ) is (among other things) the foundation of nuclear technology. A range of conservation laws exists in Quantum Physics (charge, spin, baryon number, etc).

However, apart from the conservation laws as such, the very structure of physics is conservative - as mentioned in above (in 2.2.2) physics is a hierarchic (nested) structure of consecutively appearing theories (paradigms). In physics, the future developments do not negate the past, they encompass them. The situation in artistic and architectural trends and fashions do not necessarily follow this. Impressionism was not a natural development of Classicism, but a radical departure from it. Likewise, Constructivism and Modernism in architecture did not follow from Baroque style, although some elements of Art Nouveau and Art Deco (e.g., in Chrysler building in New York or Buffalo City Hall) can be traced to earlier styles. All and all, such activities as PHA can likely enhance their overall efficiencies in adopting some lines of more rigorous physical thinking, as well as accommodate some physical concepts. A few more comments on that are below. The most efficient line here is, in our view, provided by the Complexity Theory. 


\section{Theory of Complex Systems: classical and quantum aspects}

Theory of Complex Systems, TCS, (or simply "Complexity Theory") is a recent umbrella term which has several other close (but not exactly identical) conceptual relatives, such as Chaos Theory, Nonlinear Dynamics or Computational Complexity. For the most part it studies systems which are so complicated (or so sensitive to the initial conditions) that long-term deterministic predictions about their behaviour become impractical. Contrary to reductionistic methods which see complex systems in terms of behaviour of its parts ("from parts to the whole"), TCS takes an opposite (holistic) approach. It attempts to grasp the system as a whole at once, even if it means only a sketch-like approximate description. This is what typical for the art of a political cartoons, when the entire character of some politician may be eloquently conveyed by a single successful sketch.

\subsection{Non-standard logics in decision making}

Complexity Theory has recently entered areas of business, economics and management [5-8], with Santa Fe Institute (New Mexico) being the leading inter-disciplinary centre. With scholars of a variety of experiences and persuasions, it fruitfully combines serendipity with a rigorous analysis. One of the most innovative ideas for management and decision making which came from TCS was recognition of the fallacy of the consensus. Instead of trying to achieve a (pseudo) democratic consensus ("we all agree"), the advisory and decision making bodies should strive to be at the boarder of chaos. This provides a much higher rate of idea generation and a broader platform for the choice of scenarios for actions. This strategy is an enhancement and extension of common brainstorming process, the innovation brought by the TCS is a much greater use of scientific metaphors and analogies (especially from physics). Here we outline some possible inputs of Complexity Theory for the decisions in PHA area. The following two examples, though not formally a part of TCS, are related to it in a spirit and methodology.

\subsubsection{Fuzzy logic}

Most decisions which humans make involve choices between several available options. This common every-day strategy can be enhanced and formalized by application of Fuzzy Logic (FL) which is a vivid and easy-to-use method based on the Set Theory. Microprocessors using FL are often embedded as controlling hardware into various appliances to facilitate an automated choice of operating regimes. In PHA area FL can have a two-fold use. Firstly, it is an efficient tool for the decision optimization when tough choices are to be made, e.g., in which order to restore dilapidating architecture taking into account numerous local circumstances, resource limitations, etc. Secondly, in a form of FL-hardware units it can be used for the automated control of such environmental parameters as air quality and humidity in heritage buildings, museums, etc.

Prioritization and decision making in PHA area are affected by the perceived value of objects of HA. There is no unique or pre-set scale for the relative value 
and of this-or-that piece of HA. Like with other items of human heritage, their relative importance is a social construct which is defined by many factors. Among them a public perception of HA is one of the most important. It, in turn, is affected by such straightforward fact of how a particular building presents itself in public eyes. In contacts between people the first impression (and such external factors as attire or makeup) is often significant, sometime decisive. Likewise, for HA proper night-time illumination may affect public perception of importance and value. Devices using FL are most appropriate for the task as they can (through sensors) react in real time to such factors as weather, level of air pollution (it may affect visibility), or even to the intensity of the surrounding traffic and pedestrian (tourist) flow.

\subsubsection{Quantum logic}

Quantum logic is a modified version of propositional (Aristotelian) logic in which the commutative rule falls apart. In it AB is not the same as BA. The best known example of that is Heisenberg Uncertainty Principle in quantum physics. In human affairs symmetry breaking between $\mathrm{AB}$ and $\mathrm{BA}$ happens constantly. In our decisions we often act in a non-commutative way. For a young couple a choice "career first and children later" or vice versa may lead to different outcomes. In PHA decisions a choice between, say, putting all efforts and resources in one particular building, or doing a little bit on each of several buildings, makes a difference. Appeal to methodology and spirit of Quantum Logic can elevate the quality of a decision even beyond what may be provided by FL method.

\section{Ranking principles and priority settings}

Such activities as PHA are generally complicated and diversified issues in several key ways. First, PHA is usually diversified spatially because heritage architecture (civil buildings, temples, monuments, etc) is normally spread over different localities. Secondly, significant architectural items which are located in a particular country or jurisdiction normally have historical and artistic value which is not limited to a particular locality. Preservation of major architectural landmarks is, in fact, an international issue. However, specific decision bodies dealing with PHA are often local and, correspondingly, may have limited terms of reference. Additionally, there may be conflicts of interest with local or central authorities, various ethnic and cultural groups, municipal interests, businesses and construction developments, etc. All of them may be pushing for their own priorities. On top of that, PHA is an expensive activity and hence funding problems and financial management are often affect priorities and timetables in many different ways.

\subsection{Non-uniqueness of decisions}

In practice, particular decisions about this-or-that object of PHA are not necessarily unique. There are various ways to fix the deteriorating structures, to replace missing or lost elements, to paint, to work out regulation of public access 
or adjacent patterns of traffic (e.g., designated pedestrian zones in the vicinity, etc). All the above usually imply the plethora of available options. Thus, the application of some ideas of Complexity Theory can provide a valuable conceptual leverage for the decision optimization.

\subsection{1 "Travelling Salesman" optimization}

A popular saying the best is enemy of the good is reflected in Complexity Theory in the so called "Travelling Salesman" problem. Consider a salesman who needs to visit $\mathrm{N}$ cities. In what order he should visit them to minimize the total travelling distance? For small $\mathrm{N}$ (say, for $\mathrm{N}=4$ or 5 ) the problem is trivial, but its complexity quickly increases (approximately as $\mathrm{N}$ factorial) with number of cities to visit. For $\mathrm{N}=20$ the number of solutions is about $10^{\wedge} 18$ (billion of billions). Likewise, within PHA context, a committee making priority decisions and having, say, 20 heritage buildings under its mandate face astronomical number of decision. Save of throwing a dice (which, actually, sometime may be a worthy option), the application of special algorithms invented for handling Travelling Salesman problem may provide an almost instantaneous "good enough" solution.

\subsubsection{Virtuality and visualization}

An unstoppable transition from "paper-based" to an "electronic" society has numerous radical impacts on the entire society. The implications of this revolution (which, using terminology of Thomas Kuhn [4], can be seen as a new "paradigm shift"), go far beyond a mere handling of information. Modes of operation of almost all human activities, from management to architectural design, change. Transition to a Virtual World has its reflection on PHA as well.

\subsubsection{Virtuality versus materiality}

Much of the human heritage in arts, literature and (to some degree) even architecture uses our imagination and "construction" of imaginary worlds. Such examples as the visionary architecture of American painter Thomas Cole (18011848) [e.g., The Architect's Dream, 1840, The Toledo Museum of Art], or architectural fantasies of Salvador Dali (1904-1989), or strange "impossible" architecture of Maurice Escher (1898-1972) [e.g., his famous Belvedere, 1958], or recent blockbuster screenings of J.R.R. Tolkien's The Lord of the Rings or C.S. Lewis's Narnia - all contain a plethora of virtual architectural imagery. The ideas of global simulation (e.g., recent movie Matrix) are becoming fascinating topics of public discourse [9].

In a metaphoric language it can be said that we live in a virtual world, as well as in a "real" (material) world. Our inter-human connections and interactions have always components of both of these realms. The advantage of virtual aspect is that it allows Gedankenexperiments - many decisions can be perceived using modelling and visualization prior to the step when the actual physical efforts are approved. This helps to save resources and minimize possible mistakes. 


\subsection{Simulation and symmetry breaking}

A powerful idea which recently was formulated within physics says that the most interesting phenomena often happen at the boundary between chaos and order [7, 8]. Ideas of virtuality and simulation in architectural decisions (both for "new" designs and restoration of HA) can be further augmented by the inclusion of the effects of symmetry breaking - another innovative idea of physics.

\subsubsection{Simulation in architectural context}

Exponential development of computer power (Moore's Law - doubling of computer power every 2 years) gives the notion of virtuality a new boost. Technological developments and philosophical reflections came to the point when the boundaries between "real" and "virtual" are becoming fuzzy. Simulated world becomes co-fussed with the real one to a greater and greater degree [9, 10]. At the level of quantum physics the difference between information (electronic "bits and bytes") and real (material) carriers of it becomes less obvious, sometime they are perceived as essentially almost identical. Information, like entropy becomes a category of physics.

The optimal integration of HA with the environment is another issue for PHA. For that end a prior computer simulation is the most natural tool which, at times, can be augmented by some holographic 3-dimensional images. This virtual methodology allows relatively straightforward accommodation into an architectural solution of various mathematical elements, such as Golden Mean (classical Greek proportion), Fibonacci spirals, or self-similarity at a range of scales. The latter may involve such subtle arrangements of architectural elements as periodicity at a variety of lengths (analogue of super-lattices in physics of crystals), fractal structures, or an intentional symmetry breaking.

\subsubsection{Symmetry breaking: spontaneous and intentional}

Symmetry breaking is another important physical concept with a variety of implications within physical and biological sciences, as well as outside formal boundaries of science. It has a number of interesting reflections in the world of art and architecture.

4.2.2.1 Equipartition principle This is one of the central ideas of classical statistical physics. It says that in thermodynamic equilibrium all atomic degrees of freedom have the same average energy. In human society this idea has its reflection in equal rights principle - a cornerstone of modern democracy. These principles render alleged symmetry in physical or social systems, respectively. However, good as they are in theory, these principles never work in practice in exact form. With all the best intentions, human society can never achieve full equality. All atomic motions are subjected to fluctuations which are deflection from the said equipartition principle. This breaks the above mentioned physical and social symmetry. Numerous examples of that are known in architecture.

4.2.2.2 Symmetry breaking in physics This is what makes physical (and biological) world work. In purely symmetrical systems there is no internal 
dynamics and hence there is no ascending evolution or development. In quantum physics the known example is tunnelling transition of a particle (electron) between two symmetrical potential wells. In-spite that energetically both wells are the same, any attempt to observe the particle localizes it in one or the other well. Almost all fundamental physical processes (e.g., heat conductivity) require some degree of dissymmetry between right and left. By itself heat only flows from warmer to cooler regions, not the other way around.

4.2.2.3 Symmetry breaking in architecture This is equally frequent phenomenon; many historical building have it. Some cathedrals have only one tower bell and hence look a-symmetric. Was it just a matter of accident (not enough money to build both?), or, perhaps, there was some hidden intention of the builders to leave it this way? This often remains an open question. Inside church buildings there is a symmetry breaking as well - for example, there is no right-left symmetry in the placement of icons or decorations. Same goes for many secular buildings, including HA. Thus, to respect original symmetry breaking (intentional or not) is an important outfit of PHA activities.

An interesting example of the symmetry breaking in architecture, whose roots may be traced from physics, is the Geodesic Domes designed by Buckminster Fuller (1895-1983). Its analogue in atomic physics is a molecular cluster of 60 carbon atoms in a form of a sphere. It is known as "buckyball" or "fullerene" molecule. The issue here is that 60 points cannot be symmetrically arranged on the surface of the sphere. Some inequality (symmetry breaking) is inevitable in their placing. The fact that this atomic structure has found its place in architecture creates a just another fascinating link between architecture and physics.

\subsubsection{Application for unique and unusual architecture}

Although all buildings labelled as HA are supposed to be "unique" by definition, some, using the known cliché, are "more unique than the others". Some managerial ranking is inevitable and may include complicated balance of objective and subjective factors. There some "tasteless" pieces of architecture ("architectural Kitsch"), which, while may not formally fall into the definition of $\mathrm{HA}$, are nonetheless attract public attention and interest for their originality. As there are often attempts to replace them by more standard constructions (especially, in downtowns of large cities), the preservation of these buildings presents a special challenge for PHA decisions.

\section{Conclusion}

The aim of this article is to offer some possible connections and to strengthen the bridges between physical, managerial and architectural thinking and decision making. In the spirit of interdisciplinary approach we have discussed possible inter-links between the issues of maintenance of architectural heritage and ideas coming from modern physics. As in other areas of human activity and established knowledge base (economics, ecology, demographics, etc), inter- 
breeding of methods, concepts and practices opens up a new vista and helps to shape up the efficient and productive managerial solutions.

For PHA the environmental factors play a decisive role. All numerous forms of pollution [11] have their share in this degradation and dilapidation processes [12]. Insights coming from the side of physics and science in general help to upgrade both technological and managerial sides of the PHA issue.

\section{References}

[1] Berezin, A.A., Natural evolution, human creativity and engineering design in the context of paradigms of modern physics. Design and Nature $\mathrm{IV}$, ed. C.A. Brebbia, WIT Press: Southampton, Boston, pp. 301-310, 2008.

[2] Shlain, L., Art and Physics: Parallel Visions in Space, Time \& Light. Harper Collins, New York, 1991.

[3] Schwartz, B., The Paradox of Choice: Why More is Less. Harper Collins, New York, 2005.

[4] Kuhn, T.S., The Structure of Scientific Revolution. University of Chicago Press, Chicago, 1962.

[5] Krugman, P., The Self-Organizing Economy. Blackwell, Cambridge Massachusetts, 1996.

[6] Stacey, R.D., Managing the Unknowable: strategic boundaries between order and chaos in organizations. Jossey-Bass, San Francisco, 1992.

[7] Casti, J.L., Searching for Certainty: What Scientists Can Know about the Future. William Morrow and Company, Inc. New York, 1990.

[8] Abraham, R.H., Chaos, Gaia, Eros. Harper San Francisco, Harper Collins Publishers, New York, 1994.

[9] Berezin, A.A., Simulation Argument in the Context of Ultimate Reality and Meaning, Ultimate Reality and Meaning, 29 (4), pp. 244-261, 2006.

[10] Berezin, A.A., Quantum computing and security of information systems, Safety and Security Engineering II, eds. M. Guarascio, C.A. Brebbia, F. Garcia, WIT Press: Southampton, Boston, pp. 149-159, 2007.

[11] Berezin, A.A. \& Gridin, V.V., Electromagnetic and informational pollution as a co-challenge to air pollution. Air Pollution XVI, ed. C.A. Brebbia \& J.W.S. Longhurst, WIT Press: Southampton, Boston, pp. 533-542, 2008.

[12] Weisman, A., The World Without Us. HarperCollins Publishers Ltd, Toronto, 2007. 